\title{
Microcantilever Q Control Via Capacitive Coupling
}

\section{Citation}

Huefner, Magdalena, Adam Pivonka, Jeehoon Kim, Cun Ye, Martin A. Blood-Forsythe, Martin Zech, and Jennifer E. Hoffman. 2012. Microcantilever Q control via capacitive coupling. Applied Physics Letters 101(17): 173110.

\section{Published Version}

doi:10.1063/1.4764025

\section{Permanent link}

http://nrs.harvard.edu/urn-3:HUL.InstRepos:9925387

\section{Terms of Use}

This article was downloaded from Harvard University's DASH repository, and is made available under the terms and conditions applicable to Other Posted Material, as set forth at http:// nrs.harvard.edu/urn-3:HUL.InstRepos:dash.current.terms-of-use\#LAA

\section{Share Your Story}

The Harvard community has made this article openly available.

Please share how this access benefits you. Submit a story.

Accessibility 


\title{
Microcantilever $Q$ control via capacitive coupling
}

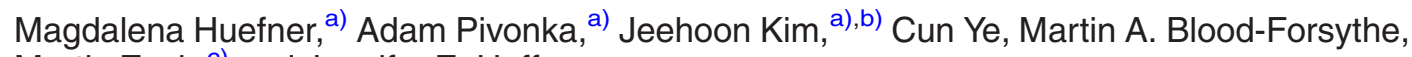 \\ Martin Zech, ${ }^{\mathrm{C})}$ and Jennifer E. Hoffman \\ Department of Physics, Harvard University, Cambridge, Massachusetts 02138, USA
}

(Received 19 July 2012; accepted 9 October 2012; published online 24 October 2012)

\begin{abstract}
We introduce a versatile method to control the quality factor $Q$ of a conducting cantilever in an atomic force microscope (AFM) via capacitive coupling to the local environment. Using this method, $Q$ may be reversibly tuned to within $\sim 10 \%$ of any desired value over several orders of magnitude. A point-mass oscillator model describes the measured effect. Our simple $Q$ control module increases the AFM functionality by allowing greater control of parameters such as scan speed and force gradient sensitivity, which we demonstrate by topographic imaging of a $\mathrm{VO}_{2}$ thin film in high vacuum. @ 2012 American Institute of Physics. [http://dx.doi.org/10.1063/1.4764025]
\end{abstract}

The atomic force microscope $(\mathrm{AFM})^{1}$ is a powerful tool for nanoscale imaging, ${ }^{2}$ manipulation, ${ }^{3,4}$ and fabrication. ${ }^{5,6}$ Despite its versatility, many externally imposed constraints limit the choice of its measurement parameters. For example, sample decay, image distortion due to thermal or piezo drift, and limited cryogen hold time all restrict the duration of data collection. Additionally, sensitive samples may place upper bounds on the imaging force.

Cantilever quality factor $(Q)$ control has been one popular method for circumventing some of these constraints. Since $Q$ is a measure of the energy dissipated in each oscillation cycle, it is proportional to the transient response time $\tau=Q /\left(\pi f_{c}\right)$, where $f_{c}$ is the cantilever resonance frequency. ${ }^{7}$ Thus, $Q$ reduction has been used to increase the bandwidth of the AFM for faster imaging in vacuum. ${ }^{8-11}$ Conversely, $Q$ enhancement can improve force sensitivity and reduce the average contact force applied to fragile samples in tapping mode. ${ }^{12-15}$ Phase contrast ${ }^{16}$ and force gradient sensitivity ${ }^{15,17,18}$ may also be increased.

Cantilever $Q$ control has previously been achieved via active damping ${ }^{19,20}$ or parametric excitation. ${ }^{21}$ Most implementations of $Q$ control are realized by means of "selfexcitation," in which a feedback signal is added directly to the original signal driving the cantilever. Active damping has also been demonstrated using state feedback control ${ }^{9,10}$ and a variety of secondary driving forces such as photothermal forces ${ }^{19}$ and radiation pressure. ${ }^{22}$

Here, we report a simple capacitive coupling technique that can be used to tune a cantilever's $Q$ by a factor of up to 260 , depending on the native $Q$ of the cantilever. We demonstrate this $Q$ control experimentally and describe the dynamics of the system with a point-mass oscillator model, which we show to be consistent with the experimentally observed behavior. Since the effect relies on capacitive coupling between the cantilever and the environment, the only requirement is that the cantilever is conductive and in close proximity to a conductive environment whose voltage $V_{s}$ can be set independently. The method can typically be employed quickly and cheaply, without modifying the AFM head.

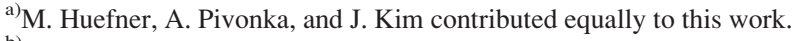

b) Present address: Los Alamos National Laboratory, National Security Science, Los Alamos, New Mexico 87545, USA.

${ }^{c}$ Present address: Attocube Systems AG, 80539 München, Germany.
}

An overview of the experimental setup is provided in Fig. 1. Light from a $1550 \mathrm{~nm}$ diode laser (Thorlabs S3FC $1550)$ enters the system via a single mode optical fiber. This light is partially reflected from the end of the cleaved fiber and partially from the cantilever, leading to an interference signal proportional to the displacement of the cantilever. A balanced photodetector (New Focus 2117) converts the interference signal to a voltage, $V_{\text {optical }}$.

A periodic drive signal $V_{d}$ deforms a "shake" piezo to oscillate the cantilever. We acquire resonance curves by sweeping the drive frequency $f_{d}$, while recording both the amplitude and phase of the cantilever's motion relative to the drive. We determine the quality factor via $Q=p f_{c} / 2$, where $p$ is the measured slope of the phase versus $f_{d}$ in the linear region centered about $f_{c}$. Consecutive $Q$ measurements under nominally identical conditions are repeatable to within $\sim 5 \%$. We have therefore rounded all reported $Q$ values to the nearest hundred.

To modify $Q$, we use a commercial amplifier (Stanford Research Systems SR560) to enhance $V_{\text {optical }}$ by a unitless gain factor $G$. We then phase shift the signal by $90^{\circ}$ relative to the cantilever displacement via a homebuilt phase shifter. The resulting voltage $V_{c}$ is applied directly to the cantilever. For periodic oscillations, $V_{c}$ is proportional to the velocity of the cantilever, and can be expressed as $V_{c}=\left(\frac{s G}{2 \pi f_{d}}\right) \dot{z}$, where $s$ is the sensitivity of the interferometer. An additional DC voltage $V_{s}$ is applied to the sample with respect to the cantilever. $G$ and $V_{s}$ may be tuned independently to change $Q$.

We demonstrate reliable $Q$ control using a variety of cantilevers and samples, detailed in Table I. All data shown in the figures were recorded under the following conditions. The AFM was at room temperature in high vacuum $\left(\sim 10^{-7}\right.$ Torr). The sample was a $\sim 100 \mathrm{~nm}$ thick $\mathrm{VO}_{2}$ film grown on a highly doped $\mathrm{Si}$ substrate. The shake piezo drive amplitude was $0.5 \mathrm{mV}$ peak-to-peak unless otherwise noted. For Figs. 2 and 3, the cantilever-sample separation was fixed at $1 \mu \mathrm{m}$, and results were found to be independent of the lateral position of the cantilever.

Figure 2 demonstrates the dependence of $Q$ on $V_{s}$ and $G$. In Figs. 2(a) and 2(b), we show the effect of varying $V_{s}$ for fixed $G=1$. Increasing $V_{s}$ simultaneously increases the height of the resonance peak and the slope of the phase plot, 


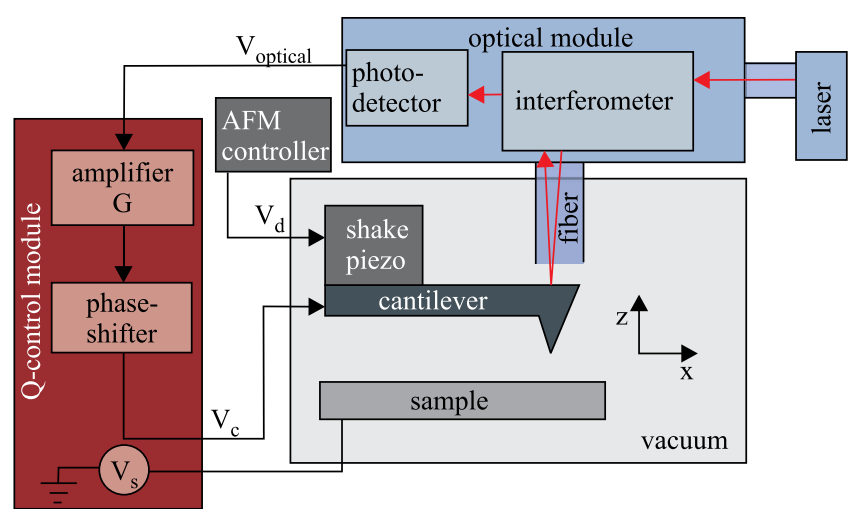

FIG. 1. Schematic diagram of the experimental setup. The $Q$ control module (red) may be added without breaking vacuum (grey) or modifying the existing optical circuitry (blue).

while decreasing $V_{s}$ has the opposite effect. Tuning $V_{s}$ therefore allows for both enhancement and reduction of $Q$. In Figs. 2(c)-2(f), we show the effect of varying $G$ for both positive and negative $V_{s}$. Increasing $G$ with negative $V_{s}$ results in $Q$ reduction [Figs. 2(c) and 2(d)], while increasing $G$ with positive $V_{s}$ leads to $Q$ enhancement [Figs. 2(e) and 2(f)].

Understanding the dependence of $Q$ on $G$ and $V_{s}$ requires modeling. Far from the sample, the equation of motion for the cantilever is

$$
m \ddot{z}+b \dot{z}+k z=F \cos \left(2 \pi f_{d} t\right)+\frac{\gamma}{2}\left(V_{c}-V_{s}\right)^{2},
$$

where $m$ is the effective mass of the cantilever, $z$ is the displacement normal to the sample surface (see Fig. 1), $b$ is the damping coefficient, $k$ is the spring constant, $F$ is the driving force amplitude (proportional to $V_{d}$ ), $t$ is the time, and $\gamma \equiv d C / d z$ is the derivative of the cantilever-environment capacitance $C$ with respect to cantilever-sample separation. ${ }^{23} V_{s}$ and $V_{c}$ are the voltages applied to the sample and cantilever, respectively. ${ }^{24}$ Substituting $V_{c}=\left(\frac{s G}{2 \pi f_{d}}\right) \dot{z} \equiv g \dot{z}$, we have

$$
m \ddot{z}+\left(b+\gamma g V_{s}\right) \dot{z}+k z=F \cos \left(2 \pi f_{d} t\right)+\frac{\gamma g^{2}}{2} \dot{z}^{2} .
$$

We ignore a constant term proportional to $V_{s}^{2}$, corresponding to a small, constant shift in the equilibrium displacement of the cantilever. ${ }^{25}$ Equation (2) differs from the standard damped, driven oscillator only in the final nonlinear term.

TABLE I. Native $Q\left(Q_{n}\right)$, as well as the minimum and maximum $Q$ for a variety of cantilevers and samples.

\begin{tabular}{lccccc}
\hline \hline Cantilever & Description & Sample & $Q_{n}$ & $Q_{\text {min }}$ & $Q_{\text {max }}$ \\
\hline A & $\mu$ masch NSC 16 & $\mathrm{VO}_{2}$ & 16000 & 3200 & 142100 \\
B & $\mu$ masch NSC 16 & $\mathrm{VO}_{2}$ & 13700 & 1400 & 92000 \\
C & $\mu$ masch NSC 18 & $\mathrm{NdFeAsO}_{1-x} F_{x}$ & 8400 & 600 & 8400 \\
D & Nanosensors & Hard drive & 100000 & 400 & 104000 \\
& TL CONT & & & & \\
E & Nanosensors & Hard drive & 21000 & 1800 & 428600 \\
& SSS QMFMR & & & & \\
\hline \hline
\end{tabular}

(a)

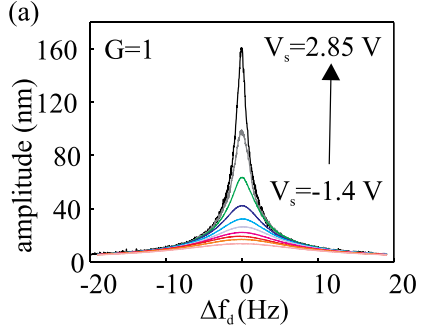

(c)

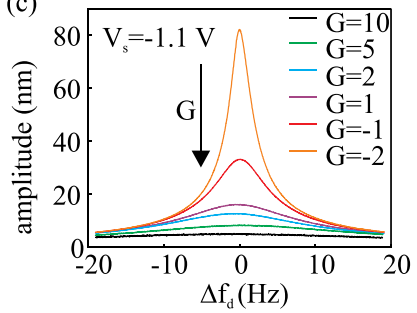

(e)

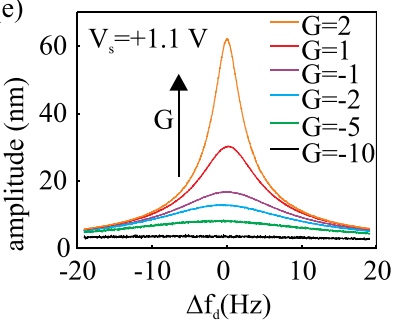

(b)

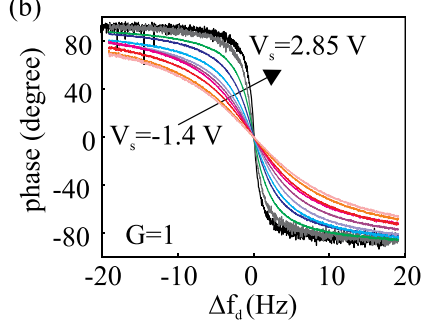

(d)

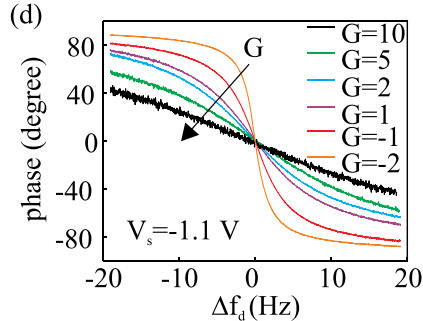

(f)

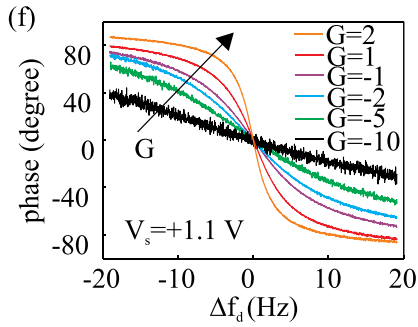

FIG. 2. Resonance curves of cantilever A for varying $V_{s}$ and $G$. The left column shows the cantilever displacement amplitude vs. frequency shift of the shake piezo relative to the resonance frequency of the cantilever $\left(\Delta f_{d} \equiv f_{d}-f_{c}\right)$. The right column shows the corresponding phase vs. $\Delta f_{d}$. The black arrows point towards increasing $V_{s}$ or $G$. (a) and (b) Resonance curves for gain $G=1$ and varying $V_{s}$. The shake piezo drive amplitude for the two traces with the highest $V_{s}$ (gray and black curves) is a factor of 5 smaller than for the other curves to avoid damaging the cantilever from large oscillation amplitudes. The oscillation amplitude shown in the plot is scaled accordingly for better comparison, leading to the artificial appearance of a larger noise level. (c) and (d) Resonance curves for $V_{s}=-1.1 \mathrm{~V}$ and varying $G$. (e) and (f) Resonance curves for $V_{s}=+1.1 \mathrm{~V}$ and varying $G$.

Note that the substitution of $g \dot{z}$ for $V_{c}$ is an approximation. The validity of the approximation is limited by our ability to adjust the phase of $V_{c}\left( \pm 10^{\circ}\right)$, by the frequency response of the phase shifter, and by the possible non-sinusoidal oscillations introduced by the nonlinear term, $\gamma g^{2} \dot{z}^{2} / 2$. However, we will show that this model describes the measured motion of the cantilever well near the resonance frequency.

In the absence of the nonlinear term,

$$
Q^{-1}=\underbrace{\frac{b}{2 \pi f_{c} m}}_{\equiv \beta}+\underbrace{\frac{\gamma s}{m\left(2 \pi f_{c}\right)^{2}}}_{\equiv \Gamma} G \cdot V_{s},
$$

where $f_{c}$ is the natural resonance frequency of the free cantilever, and we have used $f_{d} \approx f_{c}$ near resonance.

To investigate the effects of the nonlinear term, we model the motion of the cantilever using MATLAB's built-in ordinary differential equation solver, ODE45, to determine the oscillation amplitude and phase relative to $V_{d}$ from the full Eq. (2). In analogy to the experiment, $Q$ is extracted from the modeled dependence of the relative phase on the drive frequency in the linear region around resonance. ${ }^{26}$ 

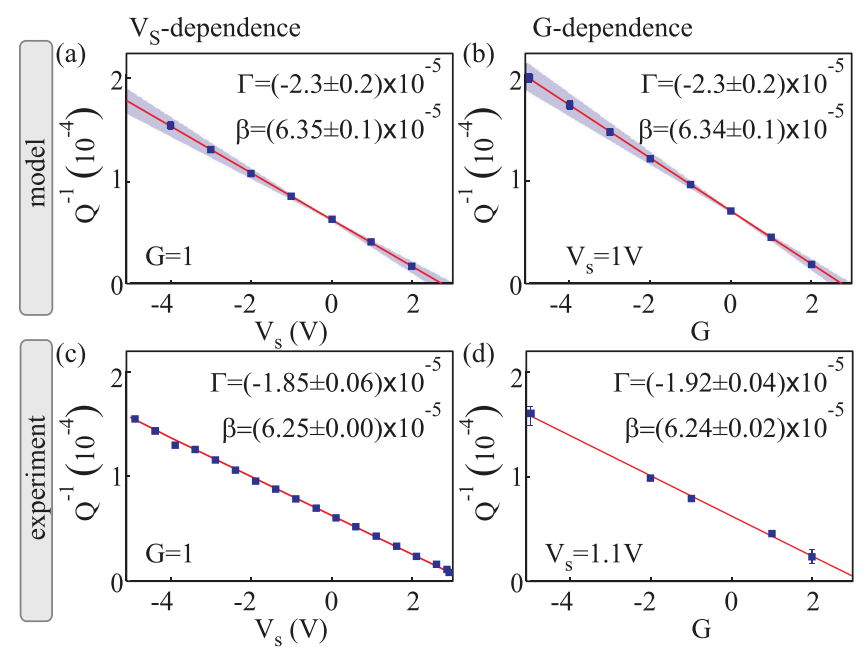

FIG. 3. Comparison of our model (top) to experimental data recorded with cantilever A (bottom). The left column shows $Q^{-1}$ vs. $V_{s}$ for fixed $G=1$; the right column shows $Q^{-1}$ vs. $G$ for fixed $V_{s}=1 \mathrm{~V}$ and $V_{s}=1.1 \mathrm{~V}$ for model and experiment, respectively. (a) and (b) The individual values for which $Q$ was modeled are shown as blue squares. The shaded area shows the range of slopes ( $\Gamma$ values) corresponding to the statistical uncertainties in the model input parameters (see Ref. 26). (c) Each blue square represents the average of $Q^{-1}$ recorded at four different lateral positions of the tip above the sample; each error bar represents the standard deviation of those four measurements. (d) Each blue square represents a single measurement of $Q^{-1}$. We recorded analogous $G$-dependent datasets for eight different $V_{s}$ values, from which we derived an average $Q^{-1}$ value for each $G$, after dividing out the known $V_{s}$-dependence. The error bars show the difference between the depicted $V_{s}=1.1 \mathrm{~V}$ dataset and the average value from all eight datasets. In all panels, linear fits to the modeled or measured data are shown in red, and the fitting parameters $\Gamma$ in $\mathrm{V}^{-1}$ and unitless $\beta$ as defined in Eq. (3) are given. The reported errors in $\Gamma$ and $\beta$ are dominated by the statistical uncertainties in the model input parameters in (a) and (b), and by the standard error of the least squares fit in (c) and (d).

We compare our experimental results with those obtained from the model by investigating the dependence of $Q^{-1}$ on both $V_{s}$ and $G$ [Fig. 3]. Experimentally, we observe that $Q^{-1}$ depends linearly on both the amplifier gain and the sample voltage for a large range of $Q$ [Figs. 3 (c) and 3(d)]. For $Q$ below about 5000, the precision with which we can determine $Q$ decreases as a result of the weak dependence of both the oscillation amplitude and the phase of the cantilever on $f_{d}$. This is apparent from the raw data displayed in Fig. 2. At the high $Q$ end of the range, typically around $\sim 150000$, we find that the oscillation can become unstable and self-reinforcing, leading to the continued oscillation of the cantilever at large amplitude even in the absence of the external driving force. In our model, this instability occurs when the damping coefficient, $b+\gamma g V_{s}$, becomes negative. Within these limits, our model reproduces the experimentally determined slope of $Q^{-1}$ to within $25 \%$ of the experimental value. This discrepancy in $\Gamma$ between model and experiment falls within two standard deviations of the measured model input parameters. There are no free parameters in the model. Most importantly, the model confirms that the non-linear term does not adversely affect our ability to set the value of $Q$.

One environment in which $Q$ control may be particularly useful is in high vacuum, where the reduction in air friction damping increases $Q$, which in turn leads to a larger time constant $\tau$. The scan speed is limited by the minimum dwell time $\tau$ required for the cantilever to adjust to the local environment at each pixel.

In Fig. 4, we demonstrate the ability to increase scan speed by reducing $Q$. The data are collected by scanning at a constant height over a $\mathrm{VO}_{2}$ sample surface while recording the frequency shift. Each image is recorded under identical conditions, including scan height, scan speed, sample bias, and oscillation amplitude. The only difference is that $Q=17300$ in (a), while in (b) we have reduced $Q$ to 5400 . In the reduced- $Q$ case, the transient response time of the cantilever is shortened, allowing faster response of the frequency shift to the local environment. For high $Q$, the slower response time of the cantilever smears the features of the surface. We have also verified that images recorded at the two values of $Q$ become nearly identical if the scan speed is reduced to allow full dwell time $\tau$ at each pixel. The main features of Fig. 4(b) are retained in these slow scans.

In summary, we demonstrate a reliable method to reversibly control $Q$ in situ via capacitive coupling of the cantilever to the environment. Modeling the behavior of the cantilever, we explain the dependence of $Q$ on the tunable parameters $G$ and $V_{s}$. The method can be implemented in any system with a conductive cantilever and a conductive sample (or sample mounted on a conductive holder). We can tune $Q$ over a typical range of approximately two orders of magnitude. We confirm that this method of $Q$ reduction may be used to improve the quality of frequency shift images for fast scans.

We thank David Weld for useful conversations. We thank Changhyun Ko and Shriram Ramanathan (Harvard University) for providing the $\mathrm{VO}_{2}$ sample used in this work. We thank Matt Tillman and Paul Canfield (Ames Laboratory) for providing the $\mathrm{NdFeAsO}_{1-\mathrm{x}} \mathrm{F}_{\mathrm{x}}$ sample used in this work. This research was supported at Harvard by the NSFfunded Nanoscale Science and Engineering Center under (a) $\mathrm{Q}=17,300$

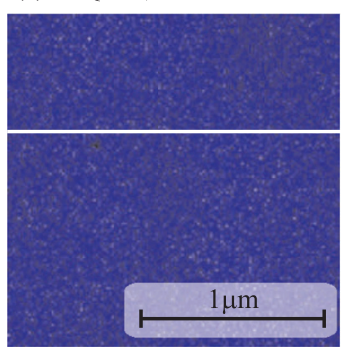

(b) $\mathrm{Q}=5,400$

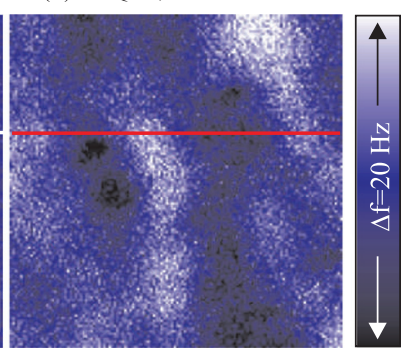

(c)

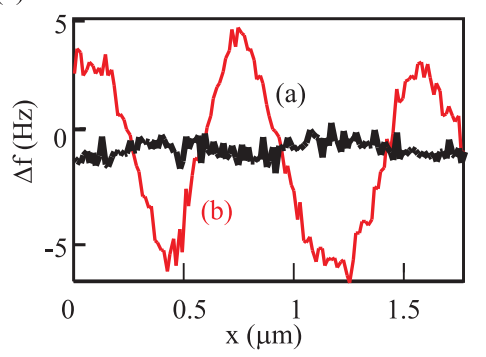

FIG. 4. Example images at $Q=17300$ (a) and reduced $Q=5400$ (b) of the same area of a $\mathrm{VO}_{2}$ film, acquired with cantilever $B$. Both images are recorded in $53 \mathrm{~s}$. The red and black traces displayed in (c) are cross sections through the data along the corresponding lines [white in (a) and red in (b)]. By reducing $Q$, the resolution is increased at unchanged scan speed. 
Grant No. PHY0117795. Adam Pivonka would like to acknowledge the support of the NSF-GRFP and the Hertz Foundation. Magdalena Huefner would like to acknowledge the support of the Deutsche Forschungsgemeinschaft (HU 1960/11).

${ }^{1}$ G. Binnig, C. F. Quate, and C. Gerber, Phys. Rev. Lett. 56, 930 (1986).

${ }^{2}$ E. Meyer, Prog. Surf. Sci. 41, 3 (1992).

${ }^{3}$ L. Roschier, J. Penttilä, M. Martin, P. Hakonen, M. Paalanen, U. Tapper,

E. I. Kauppinen, C. Journet, and P. Bernier, Appl. Phys. Lett. 75, 728 (1999).

${ }^{4}$ J. Welker and F. J. Giessibl, Science 336, 444 (2012).

${ }^{5}$ R. Held, T. Vancura, T. Heinzel, K. Ensslin, M. Holland, and W. Wegscheider, Appl. Phys. Lett. 73, 262 (1998).

${ }^{6}$ S. Masubuchi, M. Ono, K. Yoshida, K. Hirakawa, and T. Machida, Appl. Phys. Lett. 94, 082107 (2009).

${ }^{7}$ T. R. Albrecht, P. Grutter, D. Horne, and D. Rugar, J. Appl. Phys. 69, 668 (1991).

${ }^{8}$ T. Sulchek, R. Hsieh, J. Adams, G. Yaralioglu, S. Minne, C. Quate, J. Cleveland, A. Atalar, and D. Adderton, Appl. Phys. Lett. 76, 1473 (2000).

${ }^{9}$ D. Sahoo, T. De Murti, and V. Salapaka, in 44th IEEE Conference on Decision and Control, 2005 and 2005 European Control Conference, CDC-ECC'05 (2005), pp. 1185-1190.

${ }^{10}$ B. Orun, S. Necipoglu, C. Basdogan, and L. Guvenc, Rev. Sci. Instrum. 80, 063701 (2009).

${ }^{11}$ I. Gunev, A. Varol, S. Karaman, and C. Basdogan, Rev. Sci. Instrum. 78, 043707 (2007).
${ }^{12}$ J. Tamayo, A. Humphris, and M. Miles, Appl. Phys. Lett. 77, 582 (2000).

${ }^{13}$ R. Jäggi, A. Franco-Obregon, P. Studerus, and K. Ensslin, Appl. Phys. Lett. 79, 135 (2001).

${ }^{14}$ T. R. Rodríguez and R. García, Appl. Phys. Lett. 82, 4821 (2003).

${ }^{15}$ J. Kokavecz, Z. L. Horváth, and A. Mechler, Appl. Phys. Lett. 85, 3232 (2004).

${ }^{16}$ H. Hölscher, D. Ebeling, and U. D. Schwarz, J. Appl. Phys. 99, 084311 (2006).

${ }^{17}$ N. Kobayashi, Y. J. Li, Y. Naitoh, M. Kageshima, and Y. Sugawara, J. Appl. Phys. 103, 054305 (2008).

${ }^{18}$ F. Callaghan, X. Yu, and C. Mellor, Appl. Phys. Lett. 87, 214106 (2005).

${ }^{19}$ J. Mertz, O. Marti, and J. Mlynek, Appl. Phys. Lett. 62, 2344 (1993).

${ }^{20}$ U. Durig, O. Zuger, and A. Stalder, J. Appl. Phys. 72, 1778 (1992).

${ }^{21}$ D. Rugar and P. Grütter, Phys. Rev. Lett. 67, 699 (1991).

${ }^{22}$ D. M. Weld and A. Kapitulnik, Appl. Phys. Lett. 89, 164102 (2006).

${ }^{23}$ Although $\gamma \propto 1 / z$ over a large range of $z, \gamma$ can be considered to be roughly constant within the typical surface roughness of a single scan.

${ }^{24} \mathrm{We}$ are most concerned with the capacitance between the cantilever and those parts of the environment held at $V_{s}$. For the work presented here, the sample is poorly conducting $\mathrm{VO}_{2}$, so this capacitance is primarily between the cantilever and the conductive sample mount, and $\gamma<0$. In a different geometry in which $\gamma>0$, one could carry out the identical $Q$ control procedure by changing the sign of $G$.

${ }^{25}$ This is a good approximation, since the only effect of this term is a small shift of $\sim 10^{-2} \mathrm{~nm}$ in the equilibrium displacement of the cantilever.

${ }^{26}$ See supplementary material at http://dx.doi.org/10.1063/1.4764025 for a detailed description of all modeling procedures. 


\title{
Supplemental Material: \\ Microcantilever $Q$ control via capacitive coupling
}

\author{
Huefner, Pivonka, Kim et. al.
}

The motion of the cantilever is governed by Eq. 2 in the manuscript

$$
m \ddot{z}+\left(b+\gamma g V_{s}\right) \dot{z}+k z=F \cos \left(2 \pi f_{d} t\right)+\left(\frac{\gamma g^{2}}{2}\right) \dot{z}^{2}
$$

We have ignored a constant term proportional to $V_{s}^{2}$, corresponding to a small, constant shift of $\sim 10^{-2} \mathrm{~nm}$ in the equilibrium displacement of the cantilever. Eq. S1 differs from the standard damped, driven oscillator only in the final nonlinear term.

In order to effectively compare the results obtained from modeling to the experimental results, we require reasonable estimates for the model input parameters. We obtain the values of these parameters using a variety of methods. For example, our measured resonance curve with the cantilever in the native state provides the resonance frequency $f_{c}$, amplitude at resonance $\alpha$, and quality factor $Q_{n}$. We determine $\eta$, which is proportional to the capacitive coupling parameter $\gamma$, by measuring and fitting the dependence of dc cantilever deflection on $V_{s}$. The coefficient to the quadratic term in the fit is $\eta=s \gamma / 2 k$. In Fig. S1, we show example cantilever deflection data and the corresponding fit. Table SI provides an overview of the values, uncertainties, and methods of obtaining the input parameters to our model, as well as several additional parameters of possible interest.

In the main manuscript we derived the analytic dependence of $Q$ on $G$ and $V_{s}$ in the absence of the nonlinear term, Eq. 3

$$
Q^{-1}=\underbrace{\frac{b}{2 \pi f_{c} m}}_{\equiv \beta}+\underbrace{\frac{\gamma s}{m\left(2 \pi f_{c}\right)^{2}}}_{\equiv \Gamma} G \cdot V_{s} .
$$

Using the equations from Table SI for $b, m$, and $\gamma$, we can rewrite Eq. S2 as

$$
Q^{-1}=\frac{1}{Q_{n}}+2 \eta G V_{S}
$$

From Eq. S3, it becomes clear that in the absence of the nonlinear term, $Q$ depends only on the measured parameters $Q_{n}$ and $\eta$, and on the tuned parameters $V_{s}$ and $G$.

To investigate the exact behavior of the oscillation using computational methods, we cast the full Eq. S1 in a dimensionless form,

$$
\ddot{y}=\cos (B \tau)+C \dot{y}^{2}-A \dot{y}-y \text {. }
$$

In this equation, $A=\left(Q_{n}^{-1}+2 \eta G V_{s}\right), B=f_{d} / f_{c}, C=\eta \alpha s G^{2} / Q_{n}, y=z Q_{n} / \alpha$, and derivatives are taken with respect to the dimensionless time $\tau=2 \pi f_{c} t$. With this dimensionless equation, we use MATLAB's built in ordinary differential equation solver, ODE45, to model the oscillation of the cantilever as a function of the input parameters, $A, B$, and $C$. For each set of inputs, we calculate the steady-state oscillation amplitude of the cantilever and the phase of the oscillation relative to the drive signal. In Fig. S2, we plot an example of the drive signal and the position of the cantilever for a particular parameter set $\left(A=4 \times 10^{-5}, B=1, C=2.86 \times 10^{-10}\right.$, corresponding to $G=1$ and $V_{s}=1 \mathrm{~V}$ ). We determine that the oscillation amplitude is 24,900 (red arrow), and the relative phase between the curves is $(0.50 \pm 0.01) \pi$ (orange arrow).

We simulate a resonance curve measurement by sweeping the normalized frequency $B$, while holding $A$ and $C$ constant at the same values used to produce Fig. S2. In Fig. S3, we show the resonance curve and the associated phase dependence on the drive frequency obtained using the model. We determine the quality factor via $Q=p f_{c} / 2$, where $p$ is the measured slope of the phase versus $B$ in the linear region centered about resonance. From the plots in Fig. S3, we find $Q=24,500$. 
TABLE SI. Values of the model input parameters for cantilever A and the methods we use to obtain them.

\begin{tabular}{|c|c|c|c|c|}
\hline Parameter & Value & Uncertainty & Method to obtain & $\begin{array}{l}\text { Used } \\
\text { in } \\
\text { model }\end{array}$ \\
\hline$f_{c}$ & $160,970 \mathrm{~Hz}$ & $\pm 1 \mathrm{~Hz}$ & Measured from resonance sweep & yes \\
\hline$Q_{n}$ & 16,000 & \pm 160 & Calculated from $Q_{n}=p f_{c} / 2$ & yes \\
\hline$\eta$ & $-1.13 \times 10^{-5} \mathrm{~V}^{-1}$ & $\pm 0.11 \times 10^{-5} \mathrm{~V}^{-1}$ & $\begin{array}{l}\text { Quadratic fit coefficient from cantilever } \\
\text { deflection curve; reported value and un- } \\
\text { certainty are the mean and standard de- } \\
\text { viation from } 48 \text { independently measured } \\
\text { curves, a single example of which is shown } \\
\text { in Fig. S1 }\end{array}$ & yes \\
\hline$\alpha$ & $21.3 \mathrm{~nm}$ & $\pm 0.1 \mathrm{~nm}$ & Measured from resonance sweep & yes \\
\hline$s$ & $1.9 \times 10^{7} \mathrm{~V} / \mathrm{m}$ & $\pm 0.1 \times 10^{7} \mathrm{~V} / \mathrm{m}$ & $\begin{array}{l}\text { Measured by sweeping the cantilever-fiber } \\
\text { separation through a full interference } \\
\text { fringe }\end{array}$ & yes \\
\hline$k$ & $40 \mathrm{~N} / \mathrm{m}$ & $+150 \% /-63 \%$ & $\begin{array}{l}\text { Nominal value from cantilever manufac- } \\
\text { turer [see Ref. S1 for information about } \\
\text { an alternative way to derive } k \text { ] }\end{array}$ & no \\
\hline$m$ & $4 \times 10^{-11} \mathrm{~kg}$ & $+150 \% /-63 \%$ & Calculated from $m=k /\left(2 \pi f_{C}\right)^{2}$ & no \\
\hline$b$ & $2 \times 10^{-9} \mathrm{~kg} / \mathrm{s}$ & $+150 \% /-63 \%$ & Calculated from $b=k /\left(2 \pi f_{c} Q_{n}\right)$ & no \\
\hline$\gamma$ & $-5 \times 10^{-11} \mathrm{C} /(\mathrm{V} \cdot \mathrm{m})$ & $+150 \% /-63 \%$ & Calculated from $\gamma=2 \mathrm{k \eta} / \mathrm{s}$ & no \\
\hline$F$ & $50 \mathrm{pN}\left(\right.$ for $\left.V_{d}=0.5 \mathrm{mV}\right)$ & $+150 \% /-63 \%$ & Calculated from $F=\alpha k / Q_{n}$ & no \\
\hline
\end{tabular}

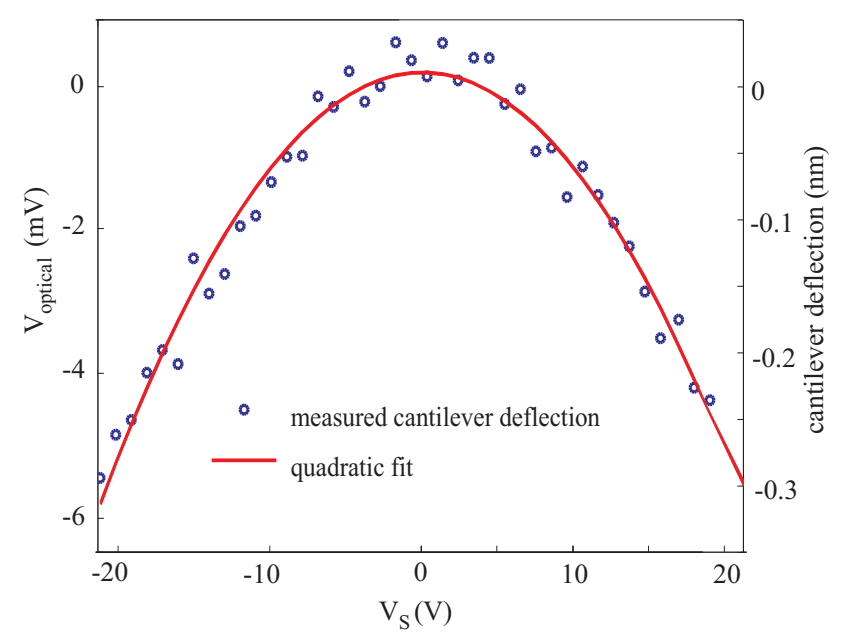

FIG. S1. Measured cantilever deflection as a function of $V_{s}$, for cantilever A. For each measured value of $V_{\text {optical }}$ (blue dot), both $V_{d}=0$ and $V_{c}=0$, i.e. there was no time-varying force from either the capacitive coupling or the shake piezo. While $V_{\text {optical }}$ is the directly measured quantity, we compute and display cantilever displacement on the right axis, using the measured value of $s$. The quadratic fit to $V_{\text {optical }}$ vs. $V_{s}$ (red line) is used to determine the coefficient to the second order term, $\eta$. 


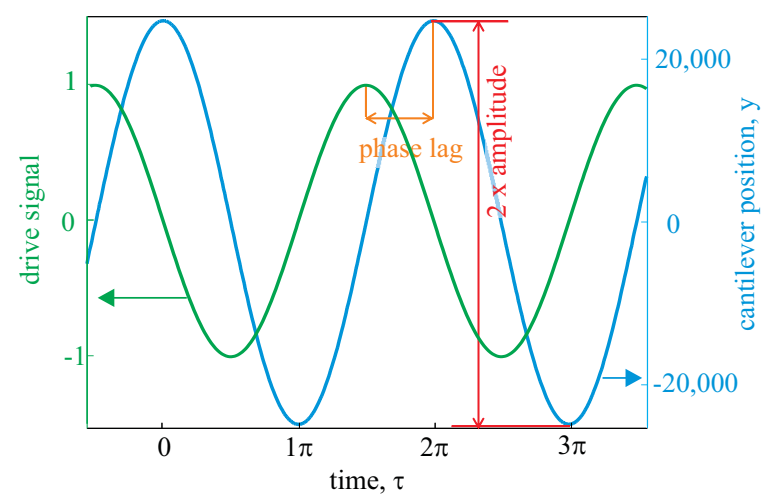

FIG. S2. Modeled drive signal and position of the cantilever versus time. The signals are plotted well into the steady-state regime.
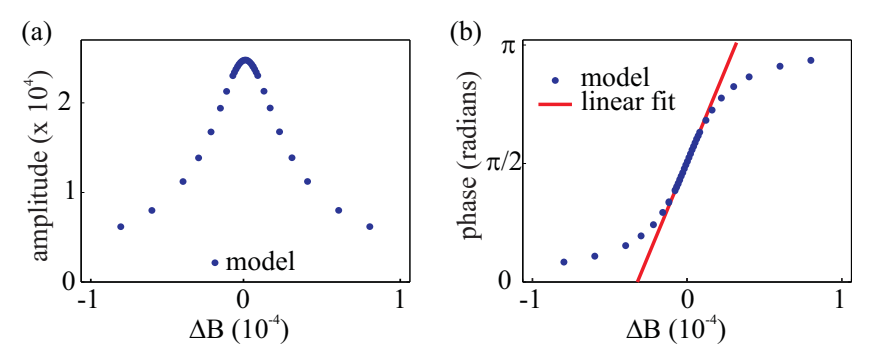

FIG. S3. Modeled cantilever oscillation amplitude (a) and relative phase (b) as a function of relative drive frequency $\Delta B=B-1$. We determine $Q$ from a linear fit to the relative phase (shown in red).

[S1]We can determine $k$ more precisely via the Sader hydrodynamic method [see Ref. S2 and Ref. S3]. Using the average dimensions (determined from scanning electron micrographs) of cantilevers originating from the same manufacturing batch as cantilever A, we obtain $k=52.2 \pm 1.7 \mathrm{~N} / \mathrm{m}$. From this value of $k$ we compute $m=5.1 \pm 0.2 \times 10^{-11} \mathrm{~kg} ; b=3.2 \pm 0.1 \times 10^{-9} \mathrm{~kg} / \mathrm{s} ; \gamma=-6.2 \pm 0.2 \times$ $10^{-11} \mathrm{C} /(\mathrm{V} \cdot \mathrm{m})$; and $F=70 \pm 2 \mathrm{pN}$.

[S2]J.E. Sader, J. W. M. Chon and P. Mulvaney, Rev. Sci. Instrum., 70, 3967 (1999).

[S3]M.L. Palacio and B. Bhushan, Crit. Rev. Solid State Mater. Sci. 35, 73 (2010). 